\title{
2012: tout, sauf la fin du monde!
}

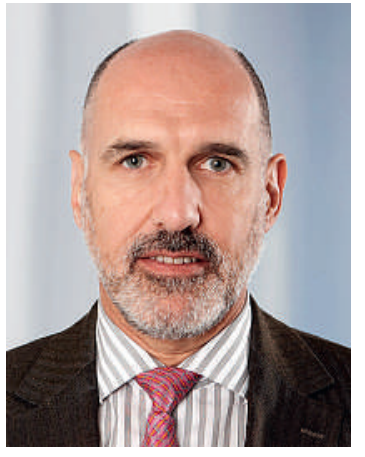

Non, 2012 ne sera pas la fin du monde. Mais ne craignez pas l'ennui pour autant: si vous aimez l'aventure, 2012 sera votre année!

Le programme politique de l'an nouveau est en effet spectaculaire, dans le domaine de la santé, et il va nous tenir occupé au maximum de nos capacités. Parce que la raison d'être de la FMH est de contribuer, autant que possible, aux meilleures conditions de travail, à la meilleure qualité de vie du corps médical en Suisse. Et cela, dans le respect de la variété des opinions et des situations de chacune et de chacun.

Je l'ai dit ici récemment, nous avons comme médecins un certain nombre de dénominateurs communs qui transcendent les différences; j'avais cité alors par exemple la place centrale du patient, de la patiente dans la médecine, la primauté de la relation thérapeutique sur les autres contingences de notre métier, l'exigence de qualité - toute cette clé de lecture de notre positionnement politique va bien sûr rester la nôtre en 2012: à quoi va-t-elle s'appliquer?

\section{Nous avons comme médecins un certain nombre de dénominateurs communs qui transcendent les différences.}

Evidemment au projet de loi sur les soins intégrés: nous allons au-devant d'une campagne de votation de plusieurs mois que nous devons absolument gagner, et nous nous y préparons déjà très activement.

Mais la liste des échéances dans lesquelles nous allons nous engager est beaucoup plus riche que cela! annoncé: le sujet est essentiel et la FMH va être interpellée là aussi.

L'introduction du nouveau financement hospitalier et des forfaits SwissDRG va nous demander beaucoup de vigilance et plusieurs études sont déjà en cours pour en suivre les effets. La révision du TARMED est aussi au programme de cette nouvelle année, vous le savez, et ce n'est pas une mince affaire - progrès et respect sont les maîtres-mots de cette opération considérable de notre service tarifaire.

\section{Nous allons au-devant d'une campagne de votation de plusieurs mois que nous devons absolument gagner.}

Et sans vouloir être exhaustif, il faut évoquer encore deux sujets au moins.

L'un est l'initiative pour une caisse maladie publique, qui va être déposée, et cela demandera que le corps médical prenne position à son égard - à voir si notre Assemblée des délégués ou la Chambre médicale pourront assumer cette décision ou si, comme cela me semble souhaitable, une nouvelle votation générale devra être organisée avant que cette initiative n'arrive devant le peuple.

Enfin, toute la question de la démographie médicale va devenir d'une actualité aiguë. La fin de la clause du besoin d'un côté et la pénurie de médecins suisses d'autre part, qui se fait de plus en plus évidente, demandent d'urgence des solutions constructives, réalistes, que nous allons tenter de formuler dans les mois qui viennent avec un groupe de travail qui est en train de se mettre sur pied.

Et je n'ai rien dit de l'arrivée d'un nouveau ministre de la santé, ni de la réélection du Comité central, qui contribueront aussi à animer 2012!

Si la valeur d'une année qui s'ouvre doit se mesurer à ce qu'elle a comme potentiel, à ce qu'elle peut apporter, alors 2012 se présente comme une année remarquable!

\section{Si la valeur d'une année qui s'ouvre doit se mesurer à ce qu'elle a comme potentiel, à ce qu'elle peut apporter, alors 2012 se présente comme une année remarquable.}

On doit citer ici l'initiative sur la Médecine de famille, qui va commencer son parcours parlementaire dans quelques semaines avant d'être soumise au peuple; il y a également plusieurs lois importantes qui demanderont notre intervention déterminée: loi sur la prévention, loi sur le dossier électronique, loi sur l'assurance-invalidité. Et bien sûr, il y a la révision de la LAMal qui veut imposer la transmission des diagnostics aux assureurs, contre laquelle un référendum a été
Non pas qu'il y ait quoi que ce soit d'acquis, pour 2012: au contraire, tout est ouvert, complètement.

Mais en fait, c'est peut-être bien à cela, finalement, qu'il faut mesurer ce qu'une nouvelle année peut apporter - et nous allons nous mettre au travail!

Avec tous les voux du Comité central à chacune et à chacun.

Dr Jacques de Haller, Président de la FMH 